\title{
Motor Sequence Learning across Multiple Sessions Is Not Facilitated by Targeting Consolidation with Posttraining tDCS in Patients with Progressive Multiple Sclerosis
}

\author{
Harald Seelmann-Eggebert, Muriel Stoppe, Florian Then Bergh, Joseph Classen $\mathbb{D}$, \\ and Jost-Julian Rumpf
}

Department of Neurology, University of Leipzig, Leipzig, Germany

Correspondence should be addressed to Jost-Julian Rumpf; jost-julian.rumpf@medizin.uni-leipzig.de

Harald Seelmann-Eggebert and Muriel Stoppe contributed equally to this work.

Received 8 December 2020; Accepted 27 January 2021; Published 9 February 2021

Academic Editor: J. Michael Wyss

Copyright (c) 2021 Harald Seelmann-Eggebert et al. This is an open access article distributed under the Creative Commons Attribution License, which permits unrestricted use, distribution, and reproduction in any medium, provided the original work is properly cited.

\begin{abstract}
Compared to relapsing-remitting multiple sclerosis (MS), progressive MS is characterized by a lack of spontaneous recovery and a poor response to pharmaceutical immunomodulatory treatment. These patients may, therefore, particularly benefit from interventions that augment training-induced plasticity of the central nervous system. In this cross-sectional double-blind cross-over pilot study, effects of transcranial direct current stimulation (tDCS) on motor sequence learning were examined across four sessions on days $1,3,5$, and 8 in 16 patients with progressive MS. Active or sham anodal tDCS of the primary motor cortex was applied immediately after each training session. Participants took part in two experiments separated by at least four weeks, which differed with respect to the type of posttraining tDCS (active or sham). While task performance across blocks of training and across sessions improved significantly in both the active and sham tDCS experiment, neither online nor offline motor learning was modulated by the type of tDCS. Accordingly, the primary endpoint (task performance on day 8) did not differ between stimulation conditions. In sum, patients with progressive MS are able to improve performance in an ecologically valid motor sequence learning task through training. However, even multisession posttraining tDCS fails to promote motor learning in progressive MS.
\end{abstract}

\section{Introduction}

Multiple sclerosis (MS) is a chronic, autoimmune inflammatory disease of the central nervous system (CNS) and the predominant cause of nontraumatic neurological disability in young adults $[1,2]$. In the majority of cases, MS begins with alternating episodes of neurological impairment and subsequent recovery referred to as relapsing-remitting MS (RRMS) [3]. Following the initial relapsing and remitting disease course, approximately $60-70 \%$ of patients with MS (pwMS) who are initially classified as patients with RRMS later convert to a secondary progressive disease course (SPMS) that is characterized by a nonrelapse-related gradual progression of neurological decline without meaningful recovery [2-5]. In addition, about $10 \%$ of pwMS already demonstrate a nonrelapse-related gradual progression of functional impairment already at the onset of the disease, which is then referred to as primary progressive MS (PPMS) $[3,4]$. Compared to RRMS, both primary and secondary progressive forms of the disease are characterized by a lack of spontaneous recovery and a poor response to pharmaceutical immunomodulatory treatment [6]. Apart from the extent of the accumulating MS-induced myelin loss and neuronal injury, MS-associated functional impairment probably depends on the ability of the central nervous system to compensate the ongoing neuronal injury [7]. One mechanism that may be able to mitigate MS-associated functional disability is the induction of CNS plasticity by repeated physical training. Given the poor response to current immunomodulatory pharmaceutical interventions and the absence 
of spontaneous recovery in PPMS and SPMS, these patients may particularly benefit from interventions that are able to augment training-induced CNS plasticity to support functional compensation for the ongoing accumulation of neuronal damage.

In the motor domain, the acquisition of new skills as well as the reacquisition of lost motor skills due to brain injury is driven by repeated skill training and evolves across online and offline learning stages that are sustained by distinct mechanisms $[8,9]$. The initial "online" learning phase during which the skill to be learned is repeatedly executed for the first time is followed by an "offline" phase that leads to a transformation of the initially labile motor memory into a more robust representation in the absence of further training $[10,11]$. Performance of the newly acquired motor skill may then be further enhanced by additional cycles of online and offline learning that ultimately enable smooth and effortless execution of the trained skill [9]. Remarkably, although overall motor task performance is compromised in pwMS compared to healthy subjects, the "online" process of motor learning (i.e., the relative skill improvement across a training session as a function of practice) seems to be relatively spared from disease-associated impairment [12-14]. Against this background, the functional impact of motor training in MS may eventually be determined by the ability to stabilize (i.e., consolidate) skill improvements that were acquired online. Facilitating posttraining offline consolidation processes may, therefore, represent a reasonable target to aid functional compensation of motor skill impairments due to MSassociated neural damage.

Noninvasive brain stimulation by transcranial direct current stimulation (tDCS) has been demonstrated to modulate neuronal excitability and behaviour in healthy human subjects as well as in patients with brain injury [15]. With respect to effects on motor learning, a large body of evidence supports that tDCS effectively promotes training-induced skill improvements across a training intervention (i.e., online learning), when task training is combined with concomitant tDCS of the primary motor cortex (M1) (e.g., [16-19]). Moreover, tDCS was also shown to beneficially interact with the offline consolidation process after training. Facilitation of consolidation may be either induced by the application of tDCS "online" during training $[20,21]$ or "offline" after termination of the training intervention [22-24]. In MS, however, neither single sessions of online tDCS during motor training [25] nor single sessions of offline application of tDCS following motor skill practice [12] facilitated online learning or offline consolidation. Given the evidence pointing to impaired susceptibility of the motor system of pwMS to a single session of tDCS, we here investigated whether multiple sessions of motor training combined with posttraining offline tDCS will specifically improve consolidation and, thus, overall motor learning across sessions in patients with progressive MS.

\section{Methods}

2.1. Ethical Standards. The study protocol was approved by the institutional ethical standards committee at the University of Leipzig (registration code, 033/17-ek) and was registered at the German Clinical Trials Register (DRKS, registered on 03 May 2018; ID: DRKS00014598). All methods were performed in accordance with the relevant guidelines and regulations and all participants provided written informed consent before study-related procedures were conducted.

2.2. Participants. Participants were recruited via the neuroimmunology outpatient clinic of the Department of Neurology at the University of Leipzig. Out of 145 patients with primary or secondary progressive MS that were prescreened for potential eligibility, finally 16 patients (PPMS, $n=10$; SPMS, $n=6)$ aged between 33 and 64 years (51.2 \pm 10.6 years, mean \pm SD, 6 females) were recruited and included in the study. Two additionally screened subjects were not included as they did not match inclusion/exclusion criteria. Disease duration (defined as years since diagnosis of progressive MS) ranged from 1 to 32 years ( $13.1 \pm 9.6$ years). Inclusion criteria comprised age between 18 and 65 years, definite diagnosis of PPMS or SPMS according to the $2010 \mathrm{McDo}-$ nald Criteria [26], a current Expanded Disability Status Scale (EDSS, [27]) score between 0 and 6.5, and right handedness according to the Edinburgh Handedness Inventory [28]. SPMS participants had to be without relapse for more than one year. Exclusion criteria included pregnancy, treatment with 4-aminopyridine within the last three months, onset of a disease modifying therapy within the last three months, other CNS diseases than progressive MS, current use of sedatives, and contraindications for tDCS (e.g., history of epileptic seizures, implanted electrical devices). None of the participants were (semi-)professional musicians or had been trained as a typist. All participants underwent a full neurological examination including the assessment of the EDSS. Fatigue was assessed using the Würzburger Fatigue Inventory for MS (WEIMuS, [29]), and symptoms of depression were assessed by the Beck Depression Inventory (BDI, [30]). The 9-hole peg test (right hand), the timed 25-foot walk test, and the spoken Symbol Digit Modalities Test (SDMT, [31]) were completed on the initial and the last session of each of both experiments to assess potential changes in fine motor function of the trained hand, walking speed, and cognitive processing.

\subsection{Experimental Design. All participants took part in two} experimental sets in a cross-over design that corresponded to two different types of posttraining tDCS intervention, i.e., posttraining sham tDCS and posttraining active tDCS. Experiments were separated by at least four weeks to minimize carry-over effects and were balanced across participants with respect to the order of the type of the posttraining tDCS intervention in the first and second experimental set. Both experimental sets were, furthermore, balanced with respect to the two different but equally difficult finger movement sequences applied in experimental sets 1 and 2 . Participants were sequentially recruited and allocated to a combination of sequence of experiments (Expt. 1=active/Expt. 2 =sham or Expt. 1=sham/Expt. 2=active tDCS) and sequence of applied finger movement sequences (Expt. $1=$ sequence 1 /Expt. 2 =sequence 2 or Expt. 1=sequence 2/Expt. $2=$ sequence 1 ) in a pseudorandomized manner using 
predefined lists to assure balancing of factors. Each of both experimental sets consisted of three training sessions on day 1 , day 3 , and day 5 , and a final assessment of motor task performance on day 8. All training sessions as well as the final assessment of motor task performance were performed in the morning between before 12 a.m. to limit the influence of potential circadian fluctuations of motor performance (Figure 1).

\subsection{Motor Sequence Learning Task. Motor sequence perfor-} mance was assessed by an adapted version of the sequential finger-tapping task introduced by Karni and colleagues [10]. For each training session as well as for the final assessments of task performance, participants were instructed to execute a five-element finger-tapping sequence on a customized four-button gaming keyboard with their right (dominant) hand. Two different but equally difficult sequences were used in experimental sets 1 and 2 (balanced across participants/order of experiments): sequence 1: "4-1-3-2-4"; sequence 2: "1-4-2-3-1" (where 1 = index finger, 2 = middle finger, $3=$ ring finger, and $4=$ little finger). Participants had to demonstrate explicit knowledge of the respective sequence prior to each training session by slowly repeating the sequence three times in a row without making a mistake. Each training session consisted of 14 consecutive blocks of sequence execution that were separated by rest periods that lasted 25 seconds (Figure 1). Prior to each training session, participants were instructed to execute the sequence as fast as possible while making as few errors as possible. Each task block was automatically terminated after 60 key taps to ensure that all participants received the same amount of training (i.e., executed the same number of finger movements). This implies that a maximum of 12 correctly executed sequences could be contained within each training block. Onset of a training block was indicated by a green fixation cross in the middle of a computer screen on a desk in front of the participants, which changed its color to red to indicate the onset of a rest block. No information on the sequence was presented to the participants during training or rest blocks. The final assessment of task performance on day 8 in both experiments consisted of only 4 blocks of the task. Participants were instructed not to practice the sequence in-between sessions to prevent confounding offline consolidation with additional task training.

\subsection{Posttraining Transcranial Direct Current Stimulation.} Posttraining active or sham tDCS was applied for 15 minutes immediately following termination of each training session on days 1, 3, and 5 of both experimental sets. Setup of the direct current stimulator (DC-Stimulator-Plus, Neuroconn, Germany) and montage of the stimulation electrodes were completed before onset of the following training session in order to start the stimulation immediately after termination of the training session. The anode $(5 \times 5 \mathrm{~cm})$ was centered at C3 (according to the 10-20 EEG system), which corresponds to the hand area of the left primary motor cortex. The cathodal electrode $(5 \times 5 \mathrm{~cm})$ was placed on the right supraorbital region (i.e., ipsilateral to the trained hand). Electrodes were covered by sponges soaked in $0.9 \%$ sodium chloride solution. At stimulation onset, direct current was increased ramp-like for 8 seconds until the final stimulation current of $1 \mathrm{~mA}$ was reached (i.e., current density $0.04 \mathrm{~mA} / \mathrm{cm}^{2}$ ). The sham stimulation procedure started identically, but the stimulation current faded out 30 seconds after reaching $1 \mathrm{~mA}$. This method was shown to ensure successful blinding of the participants $[32,33]$. The experimenter was also blind for the type of the current tDCS intervention as we used the implemented "study mode" of the stimulator that allows to use predefined codes to encode sham and active stimulation mode. For the duration of the stimulation, participants were instructed to relax and to watch a set of landscape photographs (photographs changed every 30 seconds) displayed on the computer screen in front of them.

2.6. Data Acquisition and Analysis. Timing of finger taps during sequence execution was recorded with a customized fourbutton gaming keyboard and processed using customized MATLAB (MathWorks, Natick, USA) scripts in order to extract the speed and the accuracy of sequence execution. Speed performance of sequence execution was defined as the average time that was required to perform a correct sequence within a given block of the task (average time to perform a correct sequence, TCS). Accuracy was defined as the number of correct sequences per block (i.e., a maximum of twelve correct sequences per block). Repeated measures analysis of variance (rmANOVA) with the within-subject factors block $(\mathrm{B} 1, \ldots$, and B14), training session (e.g., S1, S2, S3, and S4), and tDCS intervention (i.e., sham vs. active) was applied to speed and accuracy measures to assess effects of repeated training and the stimulation intervention on task performance. The primary outcome measure was task performance in session 4 . Secondary outcome measures were changes in the performance in the 9-hole peg test and task performance changes during online and offline stages of motor learning. Online learning across a training session was operationally defined as the difference of speed/accuracy performance between the beginning of a training session (BOT: average speed/accuracy performance of the first two blocks of the training session) and the performance at the end of that training session (EOT: average speed/accuracy of the last two blocks of the training session). Averaging across two blocks to calculate BOT and EOT was done to prevent that the online and offline learning measures were inflated or deflated due to performance fluctuations in single blocks at the beginning (e.g., warming up) or end (e.g., fatigue) of a training session. To quantify offline consolidation between sessions, we computed the difference between each individual's performance at EOT of a training session and the BOT performance of the following session. Note that online and offline performance changes were computed such that positive values indicated improved speed and accuracy performance across training and between sessions. Total online and total offline learning was then computed as the sum score of the three individual online learning measures across $\mathrm{S} 1, \mathrm{~S} 2$, and $\mathrm{S} 3$ and the three offline consolidation measures assessed between S1/S2, S2/S3, and S3/S4. A single missing TCS value for one training block of participant 14 due to zero correctly performed sequences in that block 


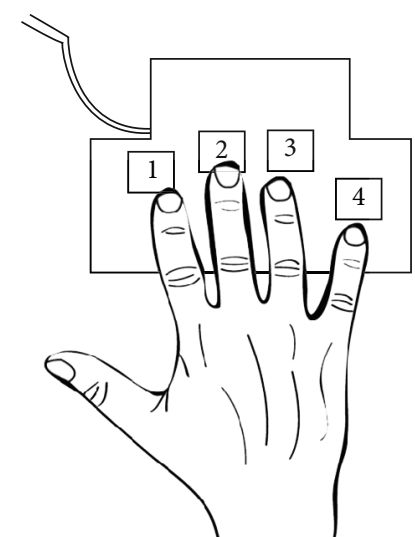

(a)
Sequence 1

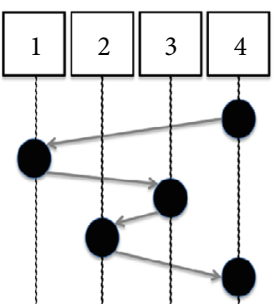

Sequence 2

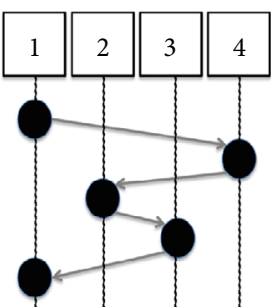

(b)

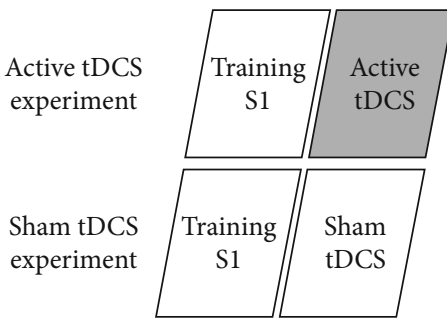

Day 1

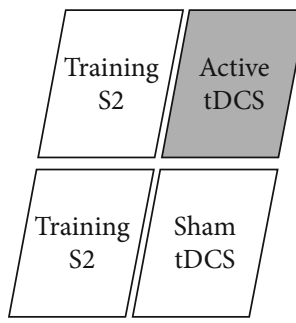

Day 3

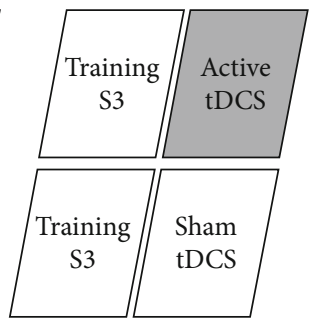

Day 5
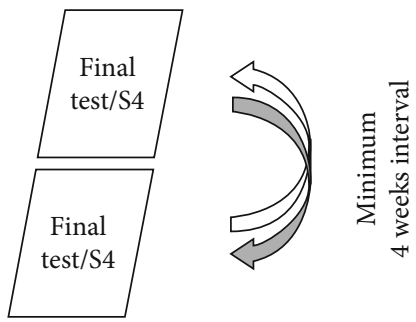

(c)

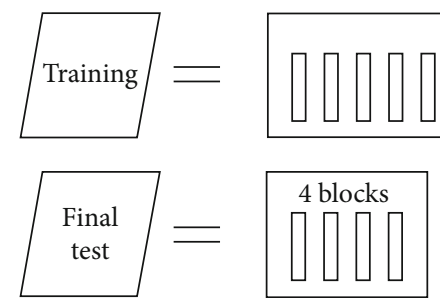

Day 8

(d)

FIgURE 1: Experimental design. (a) The explicit motor sequence learning task was executed on a four-button gaming keyboard with the right hand. (b) Participants practiced either one of two equally difficult sequences in the active tDCS experiment and the sham tDCS experiment (balanced in terms of order and assignment to the active or sham condition). (c) All participants took part in two experiments, which were separated by at least 4 weeks and corresponded to two types of posttraining tDCS interventions, i.e., active anodal tDCS of the left primary motor cortex and sham tDCS. Each experiment encompassed three training sessions on day 1 (S1), day 3 (S2), and day 5 (S3) and a final test on day 8 (S4). Posttraining tDCS was applied with the anode placed over the left primary motor cortex and the cathode placed over the right supraorbital region. (d) Each training session consisted of 14 blocks of task training, in which each encompassed the execution of 12 sequences (i.e., 60 button presses). The final test consisted of four blocks of the task.

(session 2, block 9 of the posttraining active tDCS experiment) was replaced by the mean TCS of the previous and the following block. In case of violation of the sphericity assumption, the Greenhouse-Geisser corrections were applied. Total online and offline learning values were compared between types of the posttraining tDCS intervention using paired-sample $t$-tests. Speed and accuracy measures are reported as mean with $95 \%$ confidence interval (CI). Spearman's rank correlation coefficient was applied to assess associations of motor performance, learning, and consolidation with clinical and functional characteristics. The alpha level for the correlation analyses was set to $p<0.01$. For all other statistical tests, the alpha level was set to $p<0.05$. All statistical analyses were carried out with SPSS 25 (SPSS, Chicago, IL, USA).

\section{Results}

Demographic information and clinical characteristics of the 16 individual participants are detailed in Table 1 . All of the following analyses included data from all $(n=16)$ of these participants. There were no significant differences for any of the assessed clinical and functional characteristics between the posttraining active tDCS and sham tDCS experimental sets (paired $t$-tests, all $p>0.26$ ). Of the functional tests that were assessed at baseline and at the last session (S4), only averaged performance (mean of performance across the active tDCS and the sham tDCS experiment) in the 9-hole peg test differed significantly between baseline and S4. This was driven by faster execution at $\mathrm{S} 4$ (mean $\pm \mathrm{SD}, 27.4 \pm$ $11.7 \mathrm{~s})$ compared to baseline $(28.7 \pm 12.5 \mathrm{~s}, p=0.020)$. 


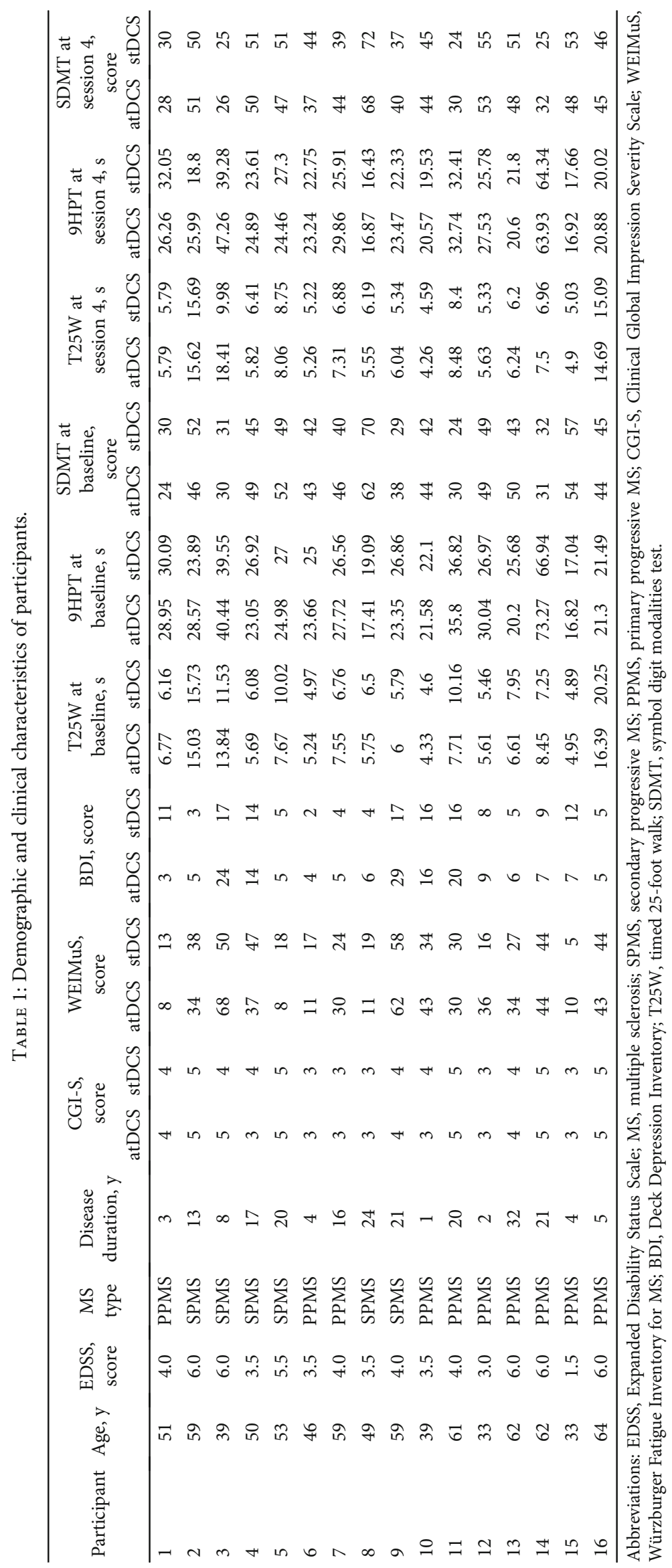


Stimulation side effects consisted in sensations described as a tingling or an itching under the stimulation electrodes. Eight of the 16 participants were able to correctly identify the order of the sham and active tDCS intervention experiments after completion of both experimental sets suggesting successful blinding.

3.1. No Modulation of Overall Motor Learning by Posttraining tDCS. Overall motor learning was assessed by comparing mean task performance in terms of speed and accuracy in session 4 (day 8), which was preceded by either 3 training sessions (on day 1, 3, and 5) combined with posttraining sham or active tDCS of M1. rmANOVA applied to mean speed performance in session 4 (mean TCS across the 4 blocks of the task) showed no significant main effect of the within-subject factor Intervention $\left(F_{(1,15)}=0.974, p=0.339\right)$ indicating that overall motor learning was not modulated by posttraining tDCS. Average TCS across blocks in session 4 amounted to $1982 \mathrm{~ms}$ (CI 1561-2402) when prior training sessions were followed by posttraining sham tDCS and to 2126 ms (CI 1498-2754) when task training was combined with posttraining active tDCS of M1.

Similar results were obtained with respect to accuracy of task performance during session 4. Mean number of correct sequences per block (maximum 12) in session 4 amounted to 11.59 (sham tDCS, CI 11.39-11.80) and 11.41 (active tDCS, CI 10.86-11.96) and did not differ significantly between stimulation conditions (Intervention: $F_{(1,15}=0.622$, $p=0.443$ ). This, collectively, indicates no beneficial effect of repeated sessions of task training combined with posttraining anodal tDCS of M1 on motor learning across sessions in patients with progressive MS.

3.2. Online Learning during Training Sessions-Speed. rmANOVA conducted on the speed measure (TCS) with the within-subject factors Block (B1, ..., and B14), Session (training sessions 1, 2, and 3), and posttraining Intervention (sham tDCS vs. active tDCS) revealed a significant main effect of Block $\left(F_{(2.5,37.6)}=5.039, p=0.007\right)$ and Session $\left(F_{(1.3,19.4)}=32.054, p<0.001\right)$ in the absence of a significant main effect of Intervention $\left(F_{(1,15)}=0.670, p=0.426\right)$ or a significant interaction of factors (all $p \geq 0.563$ ). TCS at BOT of the first training session was similar $(p=0.256)$ in the posttraining sham tDCS experiment $(2848 \mathrm{~ms}$, CI 2365-3331) and the active tDCS experiment $(3017 \mathrm{~ms}$, CI 2386-3648) and reached $2114 \mathrm{~ms}$ (CI 1608-2621, sham tDCS) and $2292 \mathrm{~ms}$ (CI 1565-3020, active tDCS; $p=0.251$ ) at EOT of the third training session (Figure 2(a)). In sum, this indicates similar online learning across blocks of practice and across training sessions irrespective of the type of the posttraining tDCS interventions. Accordingly, the overall online learning measure (sum of $\triangle B O T$ - EOT in sessions 1,2 , and 3) did not significantly differ between the posttraining sham tDCS intervention experiment (total onlinegenerated improvement: $503 \mathrm{~ms}, \mathrm{CI}-40-1045)$ and the active tDCS intervention experiment $(577 \mathrm{~ms}$, CI $5 \mathrm{~ms}-1149, p=$ 0.785; Figure 2(b)). Notably, rmANOVA further revealed a significant Block $\mathrm{x}$ Session interaction $\left(F_{(26,390)}=1.766, p=\right.$
0.013 ), which was driven by decreasing online improvements across repeated training sessions indicating asymptotic learning in training session 3 (mean (sham and active tDCS experiment) online improvement $(\triangle \mathrm{BOT}-\mathrm{EOT})$ : training 1 : $337 \mathrm{~ms}$, CI 84-591; training 2: $195 \mathrm{~ms}$, CI -34-356; training 3: $7 \mathrm{~ms}$, CI -121-136). This interpretation is further supported by the fact that, when rmANOVA was applied to the three training sessions separately, it revealed a significant main effect of Block in training sessions $1\left(F_{(3.2,47.9)=} 3.651\right.$, $p=0.017)$ and $2\left(F_{(3.1,46.7)=} 3.484, p=0.022\right)$ while the main effect of Block was not significant in training session 3

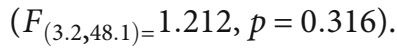

3.3. Online Learning during Training Sessions-Accuracy. A similar rmANOVA conducted on the accuracy measure (number of correct sequences per block) showed a significant main effect of Session $\left(F_{(1.3,19.7)}=10.385, p=0.002\right)$ in the absence of a significant main effect of Block $\left(F_{(3.6,53.9)}=\right.$ $1.115, p=0.356)$ and posttraining Intervention $\left(F_{(1,15)}=\right.$ $1.000, p=0.333)$. There were no significant two-way interactions for any of the factors nor a significant three-way interaction of Intervention $\mathrm{x}$ Block $\mathrm{x}$ Session (all interactions $p \geq 0.433$ ). Although mean accuracy across the first session was already high (sham and active tDCS experiments combined: 10.83, CI 10.29-11.38) and, thus, close to ceiling performance (maximum number of correct sequences per block = 12 ), it still improved gradually in the second (11.11, CI 10.66-11.57) and third training session (11.34, CI 11.0011.67), which drove the significant main effect of the factor Session (Figures 2(a) and 2(b)). Collectively, while speed performance increased during online learning and across training sessions, accuracy of task performance only gradually improved between sessions but remained stable at a high level across blocks within training sessions (i.e., no relevant speed accuracy trade-off).

Similar to speed performance, accuracy during online learning did not differ between the series of posttraining sham and active tDCS interventions. Taken together with the speed performance results, this rules out that potential effects of the posttraining tDCS intervention on offline consolidation were confounded by differences of motor engram formation during online learning.

3.4. Consolidation between Sessions-Speed. Overall speed improvements (i.e., reduction of TCS) that were generated offline between sessions (sum of differences EOT-BOT between sessions) amounted to $337 \mathrm{~ms}$ (CI -134-809) when training sessions were followed by sham tDCS and to $260 \mathrm{~ms}$ (CI -248-768) when training sessions were followed by active tDCS and did not significantly differ between stimulation conditions $(p=0.768)$. Accordingly, rmANOVA across the three single consolidation measures (between sessions $\mathrm{S} 1 / \mathrm{T} 2, \mathrm{~S} 2 / \mathrm{T} 3$, and $\mathrm{S} 3 / \mathrm{T} 4)$ revealed no significant main effect of Intervention $\left(F_{(1,15)}=0.091, p=0.768\right)$. Furthermore, rmANOVA showed no significant main effect of Session $\left(F_{(2,30)}=0.047, p=0.954\right)$ and no significant interaction of posttraining Intervention $\mathrm{x}$ Session $\left(F_{(2,30)}=1.122\right.$, $\mathrm{p}=0.339$; Figure 2(b)). This, collectively, suggests that the 

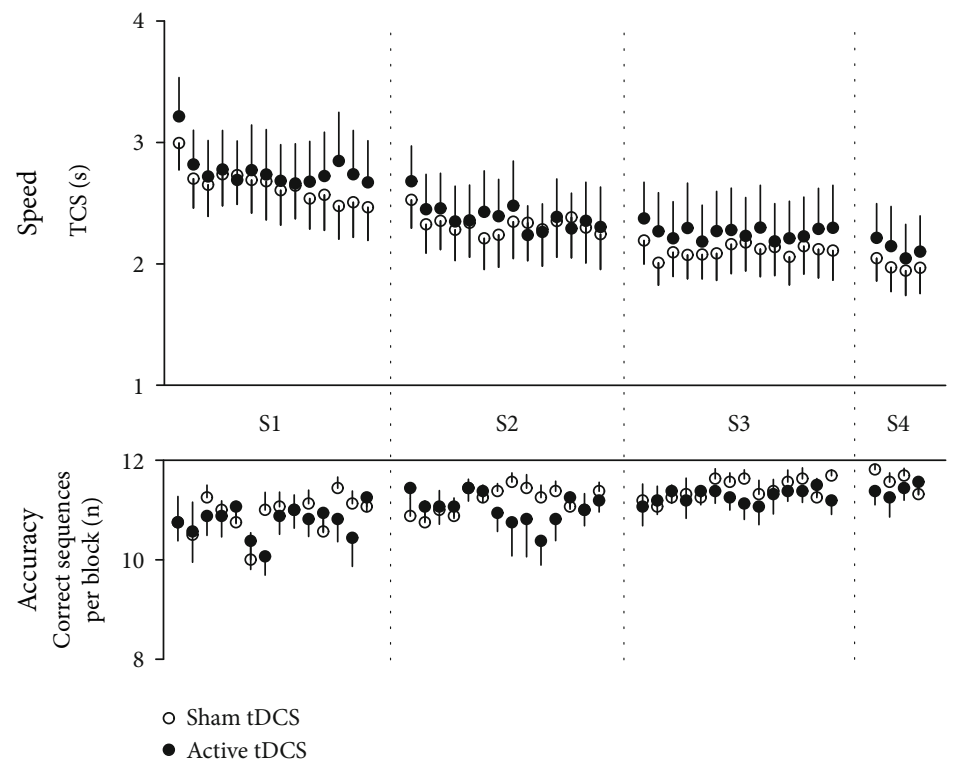

(a)

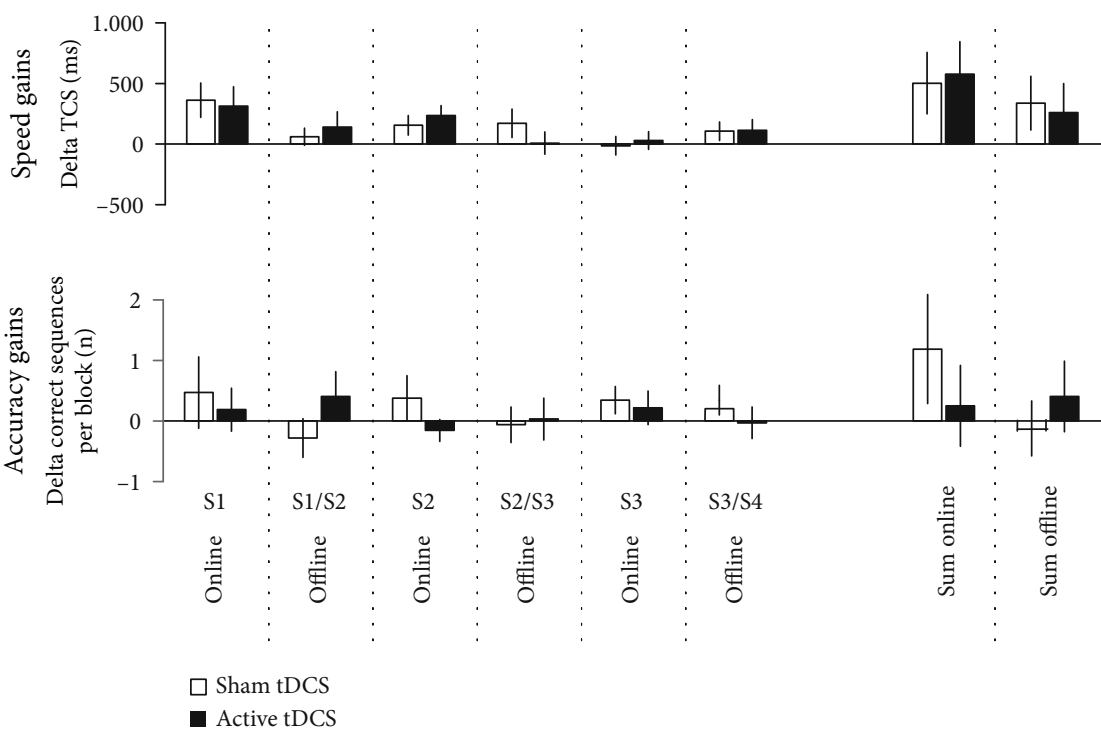

(b)

FIGURE 2: Speed and accuracy of task performance. (a) Speed (mean time to perform a correct sequence per block, TCS) and accuracy (number of correct sequences per block) of task performance across blocks of training in session 1 (S1) on day 1, session 2 (S2) on day 3, session 3 (S3) on day 5, and session 4 (S4) on day 8 of the posttraining sham and active tDCS experiments. Vertical bars represent the standard error of the mean (SEM). (b) Online and offline task performance changes. Online speed and accuracy performance changes during training sessions (S1, S2, and S3) were calculated as the difference of performance between the beginning of a training session (first two blocks) and the end of a training session (last two blocks) such that positive values indicate improvement of speed or accuracy performance across the training session. Offline performance changes between training sessions S1 and S2, S2 and S3, and S3 and S4 were assessed between the last two blocks of the previous training session and the first two blocks of the next training session such that positive values indicate offline speed and accuracy improvement of performance relative to the performance at the end of the previous session. Overall online and offline learning (sum online, sum offline) was calculated as the sum score of the three individual online (during session) and offline (between sessions) measures. Bars represent SEM.

magnitude of offline performance changes between sessions in terms of speed were comparable across the experiment and were not modulated by posttraining tDCS.

3.5. Consolidation between Sessions-Accuracy. The overall offline change between sessions (sum of between-session off- line changes) with respect to the number of correct sequences per block was comparable between posttraining sham and active stimulation $(p=0.566)$ and amounted to -0.13 correct blocks (CI -1.13-0.88) when training sessions were followed by sham tDCS and to 0.41 correct blocks (CI $-0.84-1.65$ ) when training sessions were followed by active tDCS 
(Figure 2(b)). rmANOVA across the three single consolidation measures between sessions showed no significant main effect for Intervention and Session as well as no significant interaction of factors (all $p$ values $\geq 0.275$ ). This indicates that, similar to the results for speed performance, task performance in terms of accuracy was not modulated offline between sessions by the posttraining tDCS intervention.

3.6. Correlations. Nonparametric (Spearman's rho) correlation analyses were calculated to assess associations of clinical characteristics (age, EDSS, years since diagnosis of progressive MS, BDI, and CGI-S) and functional tests (9-hole peg test, timed 25-foot walk, SDMT at baseline) with task performance measures (mean speed and accuracy performance across all blocks and sessions, sum scores of online and offline learning for speed and accuracy). As the above analyses revealed no significant differences between any of the motor sequence task performance and clinical/functional measures between the posttraining active tDCS experiment and the sham tDCS experiment, mean values of measures that were assessed twice (e.g., online learning sum score, offline learning sum score, BDI, CGI-S, 9-hole peg test, timed 25-foot walk, and SDMT) were entered into the analysis.

Not surprisingly, lower average TCS across all sessions (i.e., faster task execution) was significantly correlated with faster performance in the 9-hole peg test (rho $=0.747, p=$ 0.001 ). Moreover, lower average TCS and lower consolidation in terms of speed performance were associated with higher scores in the SDMT (rho $=-0.722, p=0.002$; rho $=$ $-0.699, p=0.003$ ), suggesting a relevant role of cognitive function for motor sequence execution and consolidation. Lower accuracy of overall task performance was significantly correlated with higher age (rho $=-0.657, p=0.006$ ) and severity of the patient's illness as rated by the CGI-S (rho $=0.645, p=0.007)$. There were no significant associations of the total online and offline learning sum scores in terms of speed and accuracy performance with other clinical characteristics (age, EDSS, years since diagnosis of progressive $\mathrm{MS}, \mathrm{BDI}$, and CGI-S scores).

\section{Discussion}

The present study showed that patients with substantial disability due to progressive MS are well capable of improving motor sequence performance across multiple training sessions but that this is not modulated by immediate posttraining anodal tDCS directed to the primary motor cortex (M1). Training-induced performance increments within training sessions were similar for the active and sham posttraining tDCS intervention sessions. This excludes that potential effects of posttraining tDCS on offline consolidation could have been confounded by differences in motor engram formation during online learning. However, we also found no relevant effect of posttraining tDCS on offline speed or accuracy performance during the consolidation phases between sessions, which were specifically targeted by the stimulation protocol. Since all participants significantly improved task performance across repeated sessions of training, failure to promote consolidation by the posttraining tDCS intervention cannot be explained by an inherent inability of the study population to improve task performance through training. Instead, it suggests that the process of offline motor consolidation was not susceptible to modulation by tDCS.

These current findings in patients with progressive MS are in line with results of a previous single-session offline tDCS study that demonstrated compromised tDCS-induced facilitation of consolidation following explicit motor sequence training in patients with relapsing-remitting MS [12]. However, those previous findings as well as the current results contrast with several studies in healthy young and older subjects that reported modulation of consolidation by offline application of tDCS after motor sequence training $[12,22-24,34]$. Moreover, in addition to the failure to promote offline consolidation in pwMS, single-session anodal tDCS directed to the primary motor cortex also failed to facilitate online motor sequence learning in pwMS when applied concurrently with task execution [25], a protocol that frequently facilitated motor sequence learning in healthy subjects in previous studies $[16,18,19,35]$. In sum, this body of evidence may point to disease-inherent properties of MS that render these patients' motor system insusceptible to the effects of $\mathrm{tDCS}$ of $\mathrm{M} 1$ with respect to online and offline motor sequence learning processes.

Results of previous studies suggested that repeated application of anodal tDCS to M1 concurrently with motor sequence training across five consecutive sessions may enhance the effects of a single-session tDCS intervention on online motor sequence learning [36] and promote overall learning by an effect on offline consolidation [21]. We are not aware of studies that investigated the application of $\mathrm{tDCS}$ concurrently (i.e., online) with multiple sessions of motor training in pwMS. Thus, there is no information on whether the absent tDCS effect on online motor sequence learning in MS observed during a single training session by Meesen and colleagues [25] may yet evolve across a multisession approach. However, current findings clearly indicate that in terms of specific facilitation of motor consolidation in pwMS, even multiple sessions of motor sequence training combined with posttraining offline tDCS are not sufficient to induce relevant effects on consolidation of training-induced speed or accuracy performance increments.

What may be the underlying pathophysiological mechanisms that render patients with relapsing-remitting [12] and progressive MS (in the current study) insusceptible to the effects of tDCS on motor sequence learning? Previous studies in healthy young subjects demonstrated that the induction of offline motor consolidation following explicit motor sequence learning was related to posttraining corticospinal excitability $[37,38]$. Moreover, it was reported that offline consolidation following motor sequence training could be facilitated by remotely applying theta burst stimulation in order to increase corticospinal excitability immediately after a motor sequence training session [38]. Facilitation of consolidation by posttraining application of anodal tDCS of M1 in healthy young $[12,22,24]$ and healthy elderly subjects [23] may have been induced by a similar mechanism (i.e., by preventing a posttraining decrease of corticospinal excitability in M1). This may imply that 
insusceptibility to the effects of posttraining tDCS on consolidation in pwMS could be related to a disease-associated impairment of modulation of corticospinal excitability by tDCS. However, previous studies showed that neither facilitation of corticospinal excitability by anodal tDCS of M1 [39] nor recruitment of LTP-like and LTD-like plasticity in M1 by transcranial magnetic stimulation differed between pwMS and healthy controls [7, 14]. This body of evidence suggests that the prerequisites to induce regional plasticity or enhance local use-dependent plasticity in M1 by noninvasive brain stimulation techniques are fundamentally intact in pwMS. However, besides local plasticity, online and offline stages of motor sequence learning rely on the dynamic recruitment of nodes of a distributed motor learning network that includes M1, the premotor cortex, the supplementary motor area, the cerebellum, basal ganglia, the hippocampus, and parietal cortical areas [40-45]. A recent fMRI study showed that alterations of sequence-specific motor learning in young healthy subjects induced by cerebellar tDCS were related to altered activity in $\mathrm{M} 1$, the cerebellum, the inferior frontal gyrus, and the right parietal lobule [46] suggesting that tDCS facilitated motor sequence learning by alterations of long-range network communication. Long-range network connectivity may be compromised in pwMS by both diseaserelated white matter lesions and affection of grey matter [47]. Thus, impaired susceptibility of pwMS to effects of tDCS of M1 in terms of modulation of online and offline motor sequence learning might be associated with a diseaserelated alteration of $\mathrm{tDCS}$-induced motor learning network recruitment that underlies the beneficial effects of tDCS on motor sequence learning in healthy subjects. However, as a limitation of our study (besides the relatively low number of participants), disease-specific deficits of pwMS in terms of susceptibility to tDCS effects on motor learning can only be inferred from the above body of evidence in healthy subjects as no healthy control group was collected in our study.

We found that fine motor hand function as assessed by the 9-hole peg test improved significantly in pwMS from baseline to the assessment at day eight, suggesting some degree of generalization of the performance improvement in the motor sequence task acquired across repeated training sessions. As this effect was specific for the 9-hole peg test and not seen for the 25-foot walk test or the SDMT (assessing working memory and executive function), one might speculate that training-induced improvements of motor sequence performance specifically generalized to another fine motor hand function. However, in terms of our primary goal to facilitate the success of repeated motor sequence training by promoting the offline consolidation phase with noninvasive brain stimulation, posttraining anodal offline tDCS of M1 failed to modulate offline processing of training-induced performance increments.

\section{Conclusions}

Our findings demonstrate that posttraining anodal offline tDCS of M1 failed to improve offline consolidation even when applied over multiple sessions. This result implicates that pwMS lack regional susceptibility to the effects of tDCS on motor sequence learning. Future studies may target multiple brain regions to investigate whether more networkoriented stimulation protocols can address the dysfunctional long-range network connectivity in pwMS and facilitate motor learning through noninvasive brain stimulation.

\section{Data Availability}

The data used to support the findings of this study are available from the corresponding author on reasonable request.

\section{Conflicts of Interest}

All authors declare that they have no conflicts of interest that are directly or indirectly related to the current research.

\section{Authors' Contributions}

Harald Seelmann-Eggebert, University of Leipzig, has a major role in the acquisition of data, patient recruitment, analysis of data, interpretation of data, and first draft of the manuscript. Muriel Stoppe, University of Leipzig, designed and conceptualized the study, acquired data and funding, recruited patients, and reviewed and edited the manuscript. Florian Then Bergh, University of Leipzig, designed and conceptualized study, acquired funding acquisition, and reviewed and edited the manuscript. Joseph Classen, University of Leipzig, designed and conceptualized study, acquired funding, and revised the manuscript for intellectual content. Jost-Julian Rumpf, University of Leipzig, designed and conceptualized study, analyzed the data, interpreted the data, and reviewed and edited the manuscript for intellectual content. Harald Seelmann-Eggebert and Muriel Stoppe contributed equally to this work.

\section{Acknowledgments}

The current work was supported by the Kurt-Goldstein Institute (grant no. 931000161). We further acknowledge support from the German Research Foundation (DFG) and Universität Leipzig within the program of Open Access Publishing.

\section{References}

[1] S. L. Hauser and J. R. Oksenberg, "The neurobiology of multiple sclerosis: genes, inflammation, and neurodegeneration," Neuron, vol. 52, no. 1, pp. 61-76, 2006.

[2] D. S. Reich, C. F. Lucchinetti, and P. A. Calabresi, "Multiple sclerosis," The New England Journal of Medicine, vol. 378, no. 2, pp. 169-180, 2018.

[3] B. G. Weinshenker, "The natural history of multiple sclerosis: update 1998," Seminars in Neurology, vol. 18, no. 3, pp. 301307, 1998.

[4] R. Dutta and B. D. Trapp, "Relapsing and progressive forms of multiple sclerosis: insights from pathology," Current Opinion in Neurology, vol. 27, no. 3, pp. 271-278, 2014.

[5] B. D. Trapp and K. A. Nave, "Multiple sclerosis: an immune or neurodegenerative disorder?," Annual Review of Neuroscience, vol. 31, no. 1, pp. 247-269, 2008. 
[6] S. Faissner, J. R. Plemel, R. Gold, and V. W. Yong, "Progressive multiple sclerosis: from pathophysiology to therapeutic strategies," Nature Reviews Drug Discovery, vol. 18, no. 12, pp. 905922, 2019.

[7] D. Zeller and J. Classen, "Plasticity of the motor system in multiple sclerosis," Neuroscience, vol. 283, pp. 222-230, 2014.

[8] N. Censor, D. Sagi, and L. G. Cohen, "Common mechanisms of human perceptual and motor learning," Nature Reviews. Neuroscience, vol. 13, no. 9, pp. 658-664, 2012.

[9] E. Dayan and L. G. Cohen, "Neuroplasticity subserving motor skill learning," Neuron, vol. 72, no. 3, pp. 443-454, 2011.

[10] A. Karni, G. Meyer, C. Rey-Hipolito et al., "The acquisition of skilled motor performance: fast and slow experience-driven changes in primary motor cortex," Proceedings of the National Academy of Sciences of the United States of America, vol. 95, no. 3, pp. 861-868, 1998.

[11] W. Muellbacher, U. Ziemann, J. Wissel et al., "Early consolidation in human primary motor cortex," Nature, vol. 415, no. 6872, pp. 640-644, 2002.

[12] J. J. Rumpf, S. Dietrich, M. Stoppe et al., "Compromised tDCSinduced facilitation of motor consolidation in patients with multiple sclerosis," Journal of Neurology, vol. 265, no. 10, pp. 2302-2311, 2018.

[13] V. Tomassini, H. Johansen-Berg, L. Leonardi et al., "Preservation of motor skill learning in patients with multiple sclerosis," Multiple Sclerosis, vol. 17, no. 1, pp. 103-115, 2011.

[14] D. Zeller, K. aufm Kampe, A. Biller et al., "Rapid-onset central motor plasticity in multiple sclerosis," Neurology, vol. 74, no. 9, pp. 728-735, 2010.

[15] E. R. Buch, E. Santarnecchi, A. Antal et al., "Effects of tDCS on motor learning and memory formation: a consensus and critical position paper," Clinical Neurophysiology, vol. 128, no. 4, pp. 589-603, 2017.

[16] A. Antal, M. A. Nitsche, T. Z. Kincses, W. Kruse, K. P. Hoffmann, and W. Paulus, "Facilitation of visuo-motor learning by transcranial direct current stimulation of the motor and extrastriate visual areas in humans," The European Journal of Neuroscience, vol. 19, no. 10, pp. 2888-2892, 2004.

[17] F. C. Hummel, K. Heise, P. Celnik, A. Floel, C. Gerloff, and L. G. Cohen, "Facilitating skilled right hand motor function in older subjects by anodal polarization over the left primary motor cortex," Neurobiology of Aging, vol. 31, no. 12, pp. 2160-2168, 2010.

[18] M. A. Nitsche, A. Schauenburg, N. Lang et al., "Facilitation of implicit motor learning by weak transcranial direct current stimulation of the primary motor cortex in the human," Journal of Cognitive Neuroscience, vol. 15, no. 4, pp. 619-626, 2003.

[19] M. Zimerman, M. Nitsch, P. Giraux, C. Gerloff, L. G. Cohen, and F. C. Hummel, "Neuroenhancement of the aging brain: restoring skill acquisition in old subjects," Annals of Neurology, vol. 73, no. 1, pp. 10-15, 2013.

[20] J. Reis, J. T. Fischer, G. Prichard, C. Weiller, L. G. Cohen, and B. Fritsch, "Time- but not sleep-dependent consolidation of tDCS-enhanced visuomotor skills," Cerebral Cortex, vol. 25, no. 1, pp. 109-117, 2015.

[21] J. Reis, H. M. Schambra, L. G. Cohen et al., "Noninvasive cortical stimulation enhances motor skill acquisition over multiple days through an effect on consolidation," Proceedings of the National Academy of Sciences of the United States of America, vol. 106, no. 5, pp. 1590-1595, 2009.

[22] V. Krause, A. Meier, L. Dinkelbach, and B. Pollok, "Beta band transcranial alternating (tACS) and direct current stimulation
(tDCS) applied after initial learning facilitate retrieval of a motor sequence," Frontiers in Behavioral Neuroscience, vol. 10, p. 4, 2016.

[23] J. J. Rumpf, M. Wegscheider, K. Hinselmann et al., "Enhancement of motor consolidation by post-training transcranial direct current stimulation in older people," Neurobiology of Aging, vol. 49, pp. 1-8, 2017.

[24] F. Tecchio, F. Zappasodi, G. Assenza et al., "Anodal transcranial direct current stimulation enhances procedural consolidation," Journal of Neurophysiology, vol. 104, no. 2, pp. 1134-1140, 2010.

[25] R. L. Meesen, H. Thijs, D. J. Leenus, and K. Cuypers, “A single session of $1 \mathrm{~mA}$ anodal tDCS-supported motor training does not improve motor performance in patients with multiple sclerosis," Restorative Neurology and Neuroscience, vol. 32, no. 2, pp. 293-300, 2014.

[26] C. H. Polman, S. C. Reingold, B. Banwell et al., "Diagnostic criteria for multiple sclerosis: 2010 revisions to the McDonald criteria," Annals of Neurology, vol. 69, no. 2, pp. 292-302, 2011.

[27] J. F. Kurtzke, "Rating neurologic impairment in multiple sclerosis: an expanded disability status scale (EDSS)," Neurology, vol. 33, no. 11, pp. 1444-1452, 1983.

[28] R. C. Oldfield, "The assessment and analysis of handedness: the Edinburgh inventory," Neuropsychologia, vol. 9, no. 1, pp. 97-113, 1971.

[29] P. Flachenecker, G. Muller, H. Konig, H. Meissner, K. V. Toyka, and P. Rieckmann, "“Fatigue" bei Multipler Sklerose," Nervenarzt, vol. 77, no. 2, pp. 165-174, 2006.

[30] A. T. Beck, R. A. Steer, R. Ball, and W. Ranieri, "Comparison of Beck Depression Inventories-IA and -II in psychiatric outpatients," Journal of Personality Assessment, vol. 67, no. 3, pp. 588-597, 1996.

[31] A. Smith, Symbol Digit Modalities Test, Western Psychological Services, Los Angeles, 1982.

[32] P. C. Gandiga, F. C. Hummel, and L. G. Cohen, "Transcranial DC stimulation (tDCS): a tool for double-blind shamcontrolled clinical studies in brain stimulation," Clinical Neurophysiology, vol. 117, no. 4, pp. 845-850, 2006.

[33] M. A. Nitsche, L. G. Cohen, E. M. Wassermann et al., "Transcranial direct current stimulation: state of the art 2008," Brain Stimulation, vol. 1, no. 3, pp. 206-223, 2008.

[34] B. Pollok, A. Keitel, M. Foerster, G. Moshiri, K. Otto, and V. Krause, "The posterior parietal cortex mediates early offline-rather than online-motor sequence learning," Neuropsychologia, vol. 146, p. 107555, 2020.

[35] C. J. Stagg, G. Jayaram, D. Pastor, Z. T. Kincses, P. M. Matthews, and H. Johansen-Berg, "Polarity and timingdependent effects of transcranial direct current stimulation in explicit motor learning," Neuropsychologia, vol. 49, no. 5, pp. 800-804, 2011.

[36] G. Dumel, M. E. Bourassa, C. Charlebois-Plante et al., "Multisession anodal transcranial direct current stimulation induces motor cortex plasticity enhancement and motor learning generalization in an aging population," Clinical Neurophysiology, vol. 129, no. 2, pp. 494-502, 2018.

[37] E. M. Robertson, D. Z. Press, and A. Pascual-Leone, "Off-line learning and the primary motor cortex," The Journal of Neuroscience, vol. 25, no. 27, pp. 6372-6378, 2005.

[38] S. Tunovic, D. Z. Press, and E. M. Robertson, “A physiological signal that prevents motor skill improvements during consolidation," The Journal of Neuroscience, vol. 34, no. 15, pp. 5302-5310, 2014. 
[39] K. Cuypers, D. J. F. Leenus, B. Van Wijmeersch et al., "Anodal tDCS increases corticospinal output and projection strength in multiple sclerosis," Neuroscience Letters, vol. 554, pp. 151-155, 2013.

[40] G. Albouy, B. R. King, P. Maquet, and J. Doyon, "Hippocampus and striatum: dynamics and interaction during acquisition and sleep-related motor sequence memory consolidation," Hippocampus, vol. 23, no. 11, pp. 985-1004, 2013.

[41] G. Albouy, V. Sterpenich, E. Balteau et al., "Both the hippocampus and striatum are involved in consolidation of motor sequence memory," Neuron, vol. 58, no. 2, pp. 261-272, 2008.

[42] K. Debas, J. Carrier, P. Orban et al., "Brain plasticity related to the consolidation of motor sequence learning and motor adaptation," Proceedings of the National Academy of Sciences of the United States of America, vol. 107, no. 41, pp. 17839-17844, 2010.

[43] J. Doyon, V. Penhune, and L. G. Ungerleider, "Distinct contribution of the cortico-striatal and cortico-cerebellar systems to motor skill learning," Neuropsychologia, vol. 41, no. 3, pp. 252262, 2003.

[44] R. M. Hardwick, C. Rottschy, R. C. Miall, and S. B. Eickhoff, “A quantitative meta-analysis and review of motor learning in the human brain," NeuroImage, vol. 67, pp. 283-297, 2013.

[45] B. R. King, J. J. Rumpf, K. F. Heise et al., "Lateralized effects of post-learning transcranial direct current stimulation on motor memory consolidation in older adults: an fMRI investigation," NeuroImage, vol. 223, article 117323, 2020.

[46] M. Liebrand, A. Karabanov, D. Antonenko et al., "Beneficial effects of cerebellar tDCS on motor learning are associated with altered putamen-cerebellar connectivity: a simultaneous tDCS-fMRI study," NeuroImage, vol. 223, p. 117363, 2020.

[47] M. Stampanoni Bassi, L. Gilio, F. Buttari et al., "Remodeling functional connectivity in multiple sclerosis: a challenging therapeutic approach," Frontiers in Neuroscience, vol. 11, p. 710, 2017. 